\title{
Sjögren's syndrome sufferers have increased oral yeast levels despite regular dental care
}

\author{
KCM Leung ${ }^{1}$, AS McMillan', BPK Cheung ${ }^{2}$, WK Leung ${ }^{3}$ \\ ${ }^{1}$ Oral Rehabilitation, ${ }^{2}$ Oral Bio-Sciences and ${ }^{3}$ Periodontology, Faculty of Dentistry, The \\ University of Hong Kong, Hong Kong SAR, China
}

Correspondence: W. Keung Leung, Periodontology, Faculty of Dentistry, The University of Hong Kong, Room 3B39, Prince Philip Dental Hospital, 34 Hospital Road, Hong Kong SAR, China. Tel.: +852 2859 0417, Fax: +852 2858 7874, E-mail: ewkleung@hkucc.hku.hk.

Running title: Oral yeast in regularly cared Sjögren's syndrome

Keywords: Candida albicans; candidiasis, oral; dental plaque; mycology; Sjögren's syndrome; xerostomia

Date of resubmission: October 24, 2006

Oral Diseases - Manuscript Copy 


\section{Abstract}

AIM: To investigate the prevalence and quantity of oral yeasts and their association with oral candidiasis in Sjögren's Syndrome (SS) patients receiving regular dental care.

MATERIALS AND METHODS: Yeasts in oral rinse and full mouth supra-gingival plaque samples from 25 primary SS, 27 secondary SS and 29 control subjects were selectively cultured. All yeasts except single-species isolates were genotyped using pulsed field gel electrophoresis (PFGE).

RESULTS: Ten (19\%) SS sufferers had symptomless candidiasis. SS subjects had a higher prevalence $(73 \%$ vs $7 \%)$ and quantity of yeasts than controls in both oral rinse and plaque samples $(P<0.05)$. Prevalence of yeasts in plaque was associated with candidiasis regardless of denture wearing $(P \leq 0.04)$. C. albicans was the predominant yeast isolated. PFGE showed 20 (66\% of total) C. albicans isolate pairs, i.e. C. albicans species isolated from plaque and oral rinse samples of the same individual, were of closely related genetic clonal types $(P<$ $0.01)$

CONCLUSIONS: Despite effective oral hygiene, more SS subjects than controls had detectable levels of oral yeasts and their presence in supra-gingival plaque was associated with candidiasis. C. albicans colonised supra-gingival biofilm even in well-maintained SS individuals, posing a challenge to the control of oral candidiasis. 
Sjögren's syndrome (SS) is a chronic, autoimmune disease characterized by lymphocytic infiltration of the exocrine glands, especially the lacrimal and salivary glands, leading to keratoconjunctivitis sicca and xerostomia. This syndrome is subclassified into primary SS (pSS), consisting of keratoconjunctivitis sicca and xerostomia, and secondary SS (sSS) when other connective tissue diseases such as rheumatoid arthritis are also associated (Fox, 2003).

Reduction in salivary output is the oral hallmark of SS. The oral manifestations of SS include dry mouth, oral soreness and ulceration, dysphagia, dysgeusia, an increased rate of dental caries, a higher prevalence of missing teeth, salivary gland enlargement, and oral candidiasis (Baudet-Pommel et al, 1994; Leung et al, 2004; McMillan et al, 2004).

Previous studies have indicated a high rate of yeast colonization in individuals with SS detected using oral rinse (Abraham et al, 1998; Radfar et al, 2003) and tongue or palate swabs (Rhodus et al, 1997). The prevalence of clinical oral candidiasis in these subjects appears to range widely ( $0 \%$ to more than $80 \%$ ) (Tapper-Jones et al, 1980; Baudet-Pommel et al, 1994; Lundström \& Lindström, 1995; Rhodus et al, 1997; Soto-Rojas et al, 1998; Almståhl et al, 1999; Pedersen et al, 1999), most likely due to those surveyed having differing oral hygiene status and/or different criteria used to diagnose oral candidiasis. The candidiasis state is generally symptomless. A variety of yeast species have been isolated from SS using different culture techniques (Kindelan et al, 1998; Radfar et al, 2003). Most reports indicate that Candida albicans is the predominant yeast isolated, although Candida glabrata and Candida tropicalis have also been found albeit infrequently (Kindelan et al, 1998; Torres et al, 2002). A Swedish study demonstrated increased prevalence (60\% vs $25 \%$ in controls) but not proportion (\%) of C. albicans from pooled supragingival plaque samples taken from the posterior teeth of non-denture wearing pSS subjects (Almståhl et al, 1999). No report is available describing the corresponding microbiology in sSS subjects.

An earlier study by this research group reported that southern Chinese SS sufferers in 
Hong Kong had a higher DMFT than controls despite good oral hygiene (Leung et al, 2004). In addition, SS subjects had significantly more clinical signs of candidiasis-related mucosal conditions than controls ( $35 \%$ vs $17 \%)$.

The present study aimed to investigate oral yeast colonization in pSS and sSS patients who were dentally well-maintained and had good oral hygiene. Putative associations between oral yeasts isolated from oral rinse or full mouth supra-gingival plaque and oral candidiasis were also investigated. The hypothesis tested was that for pSS, sSS and control subjects under regular dental care, a similar proportion and minimal amount of yeast would be detectable from their oral rinse samples and supra-gingival plaque; and oral yeast detection would be independent of the clinical candidiasis state.

\section{Material and methods}

Subjects

SS sufferers were recruited from the Rheumatology Clinic, Faculty of Medicine, The University of Hong Kong, Queen Mary Hospital, Hong Kong SAR, China. The subjects were further classified as pSS or sSS group depending on the clinical diagnosis, based on the revised version of the European Community Diagnostic Criteria for the Classification of Sjögren's syndrome (Vitali et al, 2002). All control and SS subjects had attended regular dental review at 6 months intervals for at least 12 months before study commencement. The control group comprised medically clear patients attending the Prince Philip Dental Hospital. No subjects were under active dental treatment at the time of the study. Exclusion criteria included subjects with a history of systemic autoimmune connective tissue disease, prior radiotherapy in the head and neck region, or any concurrent condition other than SS in test subjects which might cause altered saliva flow. Participants in the control group were selected 
for similar age and gender using the age/gender characteristics of both pSS and sSS groups. All subjects in the study were dentate southern Chinese. The Faculty of Dentistry Ethics Committee, the University of Hong Kong, approved the study. Written informed consent was obtained from each participant prior to admission to the study.

\section{Oral examination}

All clinical examinations and sampling were performed by one calibrated examiner (KCML). The presence of clinical signs of oral candidiasis, i.e. angular cheilitis, erythematous lesions, and or white removable plaque, was recorded (Soto-Rojas et al, 1998). Oral hygiene was assessed using the Plaque (PI) (Löe and Silness, 1964) and Calculus Indices (CI) (Silness and Löe, 1964). Denture stomatitis in denture wearers, if any, was classified as reported by Newton (1962). In brief, the condition at the denture bearing area was classified as follows. Stage 1: pin point hyperaemia; Stage 2: diffuse hyperaemia; Stage 3: granular inflammation, i.e. the mucosa has a nodular appearance with a hyperaemic surface normally present over the entire denture-bearing area but is more commonly restricted to the central areas and, in particular, under relief areas. Denture hygiene of denture wearers was accessed by scoring the cleanliness of the fitting surface as described by Blair et al, (1995): $0=$ no visible plaque detected by scraping fitting surface with flat plastic; $1=$ no visible plaque but plaque detectable by scraping of fitting surface; 2 = plaque visible upon careful examination; $3=$ plaque deposits clearly visible; 4 = gross plaque deposits.

\section{Sialometry}

All subjects were asked to rinse their mouths for $60 \mathrm{~s}$ with $10 \mathrm{ml}$ of sterile $0.01 \mathrm{M}$ phosphate-buffered saline, $\mathrm{pH} 7.2$ (oral rinse microbial sampling; as described below) before stimulated whole saliva (SWS) sampling. SWS was then obtained as described in an earlier 
report (Leung et al, 2004). In brief, each subject was instructed to chew on a piece of sterilized silicone rubber tubing for 5 minutes and to expectorate all SWS into a sterile, pre-weighed plastic cup. All samples were collected between 9 a.m. to 12 noon. The flow rate was determined and expressed as $\mathrm{ml} / \mathrm{min}$, as described previously (Leung et al, 2004).

\section{Microbial sampling}

An oral rinse sampling protocol was used as described previously (Leung et al, 2000). Dentures, if present, were not removed during rinsing. Supra-gingival plaque samples were collected after the oral rinse. Plaque was removed with a sterile sickle scaler passing along the gingival margins of all standing teeth after air dry and cotton wool isolation. The sample was dispersed into a pre-weighed vial containing $1 \mathrm{ml}$ reduced transport fluid. Care was taken to avoid sampling plaque sub-gingivally and at sites covered by removable denture components or with open carious lesions. Full mouth supra-gingival plaque was removed and the net wet weight in grams was calculated.

\section{Culture and identification of yeast isolates}

All oral rinse and plaque samples were used neat, and vortexed for $30 \mathrm{~s}$ at maximum setting (Autovortex Mixer SA2, Stuart Scientific, London, UK). Fifty microlitre of each of the oral rinse and plaque samples were serially diluted and spiral plated (Model DU, Spiral Systems, Cincinnati, $\mathrm{OH}$ ) on to CHROMagar Candida plates (Becton Dickinson Europe, Le Pont de Claix, France), then incubated aerobically for $48 \mathrm{~h}$ at $37^{\circ} \mathrm{C}$. After incubation, plates with appropriate number of colonies that were well-separated and evenly dispersed with up to approximately 100 colony-forming units (c.f.u.) were chosen for counting. The number of yeast c.f.u. per $\mathrm{ml}$ of oral rinse or per gram wet weight of plaque was calculated. Then a stainless steel template was used to subdivide the agar into sectors with a defined area (Leung 
et al, 2005). A sector with 6-10 colonies including all different colony morphotypes observed was selected and all colonies within the sector were subcultured using Sabouraud's dextrose agar (SDA; Oxoid, Unipath Ltd, Basingstoke, Hampshire, UK) to obtain pure isolates. For samples that gave rise to $\leq 10$ yeast colonies, all colonies were subcultured. The estimated lowest detection level for yeast using the current protocol is 20 c.f.u. per $\mathrm{ml}$ oral rinse or 20 c.f.u. per full mouth plaque sample.

The yeasts were identified and speciated based on the following: colony morphology in CHROMagar and SDA, cell morphology, gram staining reaction, germ tube test; and API 20C AUX test (Leung et al, 2000). To differentiate C. albicans from C. dubliniensis, all isolates with the appropriate presumptive identity were incubated aerobically on SDA, $48 \mathrm{~h}$ at $45^{\circ} \mathrm{C}$, and on phenol red agar for $48 \mathrm{~h}$ at $26^{\circ} \mathrm{C}$ and $37^{\circ} \mathrm{C}$, respectively (Sancak et al, 2005). All plates were examined macroscopically for colony morphology and then microscopically for chlamydospore formation. Isolates from the same specimen with identical characteristics/test results based on the above identification protocol were considered as one isolate in the following experiments. Isolates that could not be identified in this manner were further characterized by MicroSeq D2 LSU rDNA fungal identification system (Applied Biosystems, Foster City, CA, USA). Yeast strains, of the same species, isolated from both oral rinse and plaque samples of an individual with identical biochemical or API 20C AUX characteristics, or identical MicroSeq D2 LSU rDNA fungal identification result, i.e. of same presumptive biotype or genetic identification, were considered to be an isolate pair. Their genetic relatedness would then be tested by PFGE.

\section{Total anaerobic plaque bacteria count}

All plaque samples were vortexed for $20 \mathrm{~s}$ at maximum setting (Autovortex Mixer) and then serially diluted in 10-fold increments up to a ten thousand fold in trypticase soy broth (TSB). 
The diluted samples were plated onto enriched Columbia blood agar (CBABS, Columbia agar base with 5\% defibrinated horse blood, $0.0005 \%$ hemin and $0.00005 \%$ Vitamin K) (Leung et al, 2005) using a Spiroplater. CBABS plates were incubated in an anaerobic chamber (Forma Scientific Inc, Marietta, $\mathrm{OH}$ ) under an atmosphere of $10 \% \mathrm{CO}_{2}, 10 \% \mathrm{H}_{2}$, $80 \% \mathrm{~N}_{2}$ for $5-7$ days at $37^{\circ} \mathrm{C}$.

CBABS plates with appropriate number of colonies that were well separated and evenly dispersed were selected, i.e. plates with 30 - 300 c.f.u., were chosen for counting. With reference to the counts, inoculum size and the dilution factor, the total anaerobic viable counts (TVC) per gram wet weight supra-gingival plaque were measured. The percentage proportion of yeast over the corresponding total anaerobic viable count was then calculated.

\section{Chromosomal analysis of yeast isolates by PFGE}

All yeast isolates were subjected to PFGE analysis. Preparation of the yeast DNA was performed according to the instruction manual of CHEF Genomic DNA Plug kits (Bio-Rad Laboratories, Hercules, CA, USA).

The chromosomes of the yeast isolates were separated employing the contour-clamped homogeneous electric field (CHEF) technique using the CHEF-DR III Variable angle system (Bio-Rad Laboratories, Hercules, Calif.) in $0.5 \times \mathrm{TBE}$ buffer at $10^{\circ} \mathrm{C}$ as described earlier with modifications (Leung et al, 2000). The electrophoretic conditions used were as follows: two state mode with linear ramping factors; run time, $72 \mathrm{~h}$; initial and final switching times, 3 min and $17 \mathrm{~min}$, respectively; constant voltage of $3 \mathrm{~V} / \mathrm{cm}$, switching angle, 106 degrees. Hansenula wingei and Saccharomyces cerevisiae chromosomes (Bio-Rad) were used as the molecular size standards. The resulting gel was stained with $0.5 \mu \mathrm{g} / \mathrm{ml}$ ethidium bromide (Sigma) in distilled water for $15 \mathrm{~min}$ and then destained in distilled water for $3 \mathrm{~h}$. DNA bands were visualized and photographed under UV transillumination. The PFGE profiles of the 
yeast isolates were confirmed by repeating the assay two more times on separate occasions.

\section{Dendrogram analysis of yeast isolates}

PFGE pattern of all yeast strains group under the same species, except those of a single isolate was analysed using the Dendron ${ }^{\circledR}$ 3.0 Program (Soll-tech, Oakdale, CA, USA). The unweighed pair group method with arithmetic means (UPGMA) was used for clustering of the isolates on the dendrogam. The method was applied to $61,5,4,3$ and 2 strains each of $C$. albicans, C. parapsilosis, C. tropicalis, S. cerevisiae, and C. dubliniensis, C. glabrata, or Trichosporon mucoides, respectively. One strain each of C. guilliermondii, C. krusei, and C. lusitaniae, C. pelliculosa, C. pseudointermedia isolate from control or sSS oral rinse samples, respectively was not analyzed. Closely related genetic clusters of individual yeast species were identified and the genetic similarities were measured. Isolate pairs were considered to be of the related genetic clonal type when $\mathrm{S}_{\mathrm{AB}} \geq 0.85$ (Clemons et al, 1997).

\section{Statistical analysis}

The demographic and microbiological parameters were analyzed and compared among the pSS, sSS and control groups. Differences in categorical variables among the three groups were tested by $\chi^{2}$ test or Fisher's exact test whichever appropriate. One-way ANOVA, unpaired t, or Kruskal-Wallis tests, whichever appropriate, was performed for evaluating differences in continuous variables among the three groups. Multiple comparisons were carried out with Bonferroni correction of $P$-values. Spearman's rank correlation coefficient was used to explore associations between parameters of interest. A 5\% level of significance was used for the statistical tests. All data were analyzed using SPSS for Windows 12.0.1. 


\section{Results}

\section{Participants}

The study population consisted of 25 pSS (1 male; mean age 50.7, range 33-74) and 27 sSS ( 1 male; mean age 42.7, range 26-66) patients. The mean time since diagnosis was for 7.2 and 3.9 years respectively for pSS and sSS. The control group had 29 participants ( 2 men; mean age 43.9, range 27-75). All except one woman in the control group were non-smokers. There was no age-related difference among the three groups $(P>0.05)$. Duration of last dental recall visit was from within 5 months- 2 weeks to 6 months- 2 weeks for all participants. All subjects were partially or fully dentate. Other than fluoridated tooth-paste, no control or SS subject used homecare fluoride products. None of the SS patients were taking medication for dry mouth symptoms. Seven (28\%) pSS and eight (30\%) sSS patients were taking hydroxychloroquine for management of extra-glandular manifestation of SS. None of the patients were receiving anticholinergic therapeutic agents. Eighteen sSS patients had SLE, four had RA, two had sicca symptoms, two had SLE-like disease one had autoimmune hepatitis.

\section{Sialometric parameters}

SS sufferers had significantly lower stimulated whole saliva flow than the controls (pSS/sSS/control: $0.3 \pm 0.4 / 0.4 \pm 0.4 / 0.9 \pm 0.5 \mathrm{ml} / \mathrm{min}$, ANOVA $P<0.001 ; \mathrm{pSS} / \mathrm{sSS}<$ control, Bonferroni multiple comparison).

\section{Oral hygiene and denture status}

Among the $\mathrm{pSS} / \mathrm{sSS} /$ control subjects, 88/89/100\% tooth-sites respectively had plaque score of $\leq 1$ and $84 / 78 / 79 \%$ respectively had a calculus score of 0 (calculus free) $(P=0.111$ and 0.884 respectively, $\chi^{2}$ test), indicating on average a satisfactory level of oral cleanliness. 
There was a statistical significant difference in number of removable denture wearers between groups ( $P=0.001$, Fisher's exact test, control vs total SS). Eight pSS subjects $(32 \%)$ wore at least one removable denture, no sSS subjects wore removable dentures whereas six $(22 \%)$ of them presented with fixed prostheses in at least one jaw. Two controls subjects (7\%) wore a single removable partial denture and one had a mandibular fixed prosthesis. Regarding denture hygiene (Blair et al, 1995), 4 pSS subjects had score 0, 3 had score 1, 1 had score 2 while the 2 control denture wearers had score 0 . None of the subjects surveyed had denture stomatitis.

\section{Soft tissue}

Prevalence of oral candidiasis based on clinical signs among the 3 groups was as shown (Table 1). None of those affected experienced symptoms. There was a statistical significant difference in the prevalence of oral candidiasis between the control and SS subjects (Table 1).

\section{Isolation of oral yeasts}

The mean wet weight of full-mouth supra-gingival plaque sampled was $10.4 \pm 32.0 \mathrm{mg}$ (range: $0.3-295.2 \mathrm{mg}$ ). The prevalence and quantity of yeast colonization are summarized in Table 1. More pSS (84\%) and sSS (63\%) sufferers than control subjects (7\%) had oral yeast detectable from either oral rinse or full mouth plaque samples $\left(P<0.0001, \chi^{2}\right.$ test $)$. For SS cases as a whole, there was a yeast prevalence of $56 \%$ for both oral rinse and plaque and $73 \%$ for yeast in either oral rinse or plaque in SS subjects. Fifteen SS subjects had no detectable level of yeast in plaque or oral rinse samples. Primary SS subjects also had a significantly higher mean yeast count in both samples compared with sSS subjects and controls $(P<$ 0.001). All SS patients who wore dentures had oral yeast detectable in both samples. A positive correlation was detected between oral colonization by yeast and removable denture 
wearing $\left(\mathrm{r}_{\mathrm{s}}=0.334, P=0.004\right)$. When non-denture wearers were considered, a similar pattern of more yeasts detectable from oral rinse or full mouth supra-gingival plaque of SS than control subjects was observed $(\mathrm{pSS} / \mathrm{sSS} /$ control; $\mathrm{n}=17 / 27 / 27$; mean oral rinse yeast count: 242.4 $\pm 351.3 / 155.0 \pm 327.2 / 0.7 \pm 3.9$ c.f.u. $/ \mathrm{ml}$; mean full mouth supra-gingival plaque yeast count $\left[\times 10^{6}\right]: 0.0 \pm 0.0 / 2.3 \pm 5.0 / 0.4 \pm 0.8$ c.f.u./g wet weight; mean percentage proportion yeast per total viable anaerobic count $\left[\times 10^{-3}\right]: 0.0 \pm 0.0 / 0.3 \pm 0.7 / 1.2 \pm 5.2 \%, \quad P<0.001$, Kruskal-Wallis test). Four pSS, 10 sSS and 27 control subjects did not carry yeasts above detectable level in their oral rinse or full-mouth plaque samples.

A total of 84 yeast strains were isolated. Six pSS and six sSS sufferers harboured more than 1 species of yeast, and 3 species of yeast were recovered from two sSS sufferers. The most predominant yeast isolated was $C$. albicans, with 28 and 33 strains isolated from oral rinse and full mouth supra-gingival plaque samples, respectively. Growth characteristics, API 20C AUX test, and chlamydospores production on phenol red agar confirmed the identity for all C. dubliniensis and all except 5 C. albicans isolates which had API 20C AUX profile as $C$. albicans but did not grow under $45^{\circ} \mathrm{C}$ and formed chlamydospores on phenol red agar at $26^{\circ} \mathrm{C}$. The final C. albicans identity of these isolates was confirmed by MicroSeq D2 LSU rDNA fungal identification. The various types of yeasts cultivable from both samples are shown in Table 2. No statistically significant difference was detected between the mean c.f.u. of the various yeast species among the three groups apart from $C$. albicans $(P<0.001)$.

\section{Oral yeast isolation and candidiasis}

Seven out of 10 SS subjects with candidiasis had yeast detectable from both oral rinse and plaque samples. One sSS subject with candidiasis had yeast detectable from supra-gingival plaque while the remaining two affected SS subjects did not have any detectable yeast from plaque or oral rinse samples. They were all non-denture wearers. Among the two with no 
yeasts detectable, one subject (pSS) had no intra-oral mucosal lesions except mild angular cheilitis. The other sSS subjects had clinical oral mucosa manifestations of candidasis. Sjögren's syndrome subjects with oral candidiasis tended to have higher mean yeast and $C$. albicans counts in both oral rinse and plaque samples than those who did not, although the difference was not statistically significant (SS subjects with candidasis vs without candidiasis: mean yeast/C. albicans in oral rinse, $426 / 418 \mathrm{vs} 361 / 355 \mathrm{cfu} / \mathrm{ml}$; mean yeast $/ C$. albicans in plaque, $2.7 \times 10^{6} / 2.7 \times 10^{6}$ vs $\left.4.3 \times 10^{5} / 4.2 \times 10^{5} \mathrm{cfu} / \mathrm{gm}\right)$. However, a positive correlation was observed between the prevalence of oral candidiasis and the prevalence of yeast isolated in plaque but not in the oral rinse $\left(\mathrm{r}_{\mathrm{s}}=0.269, P=0.02\right.$ and $\mathrm{r}_{\mathrm{s}}=0.223, P=0.082$, respectively). Despite the high isolation rate of yeast associated with removable denture wearing (Table 1), there was no correlation between oral candidiasis prevalence and the appliance usage among subjects in all three groups $(P=0.604)$. When denture wearers were excluded from the data analysis, subjects with oral candidiasis had higher but not statistically significant mean yeast or C. albicans counts in both oral rinse and plaque samples (mean yeast/C. albicans in oral rinse, $467 / 460$ vs $73 / 68 \mathrm{cfu} / \mathrm{ml}$; mean yeast $/ C$. albicans in plaque, $3.3 \times 10^{6} / 3.2 \times 10^{6} \mathrm{vs}$ $\left.3.8 \times 10^{5} / 3.8 \times 10^{5} \mathrm{cfu} / \mathrm{gm}\right)$. Nonetheless, positive correlations were observed between the prevalence of oral candidiasis and the prevalence of yeast isolated from oral rinse or plaque in non-denture wearers $\left(\mathrm{r}_{\mathrm{s}} \geq 0.257, P \leq 0.044\right)$.

\section{PFGE analysis of yeast isolates from SS subjects}

All yeast isolates except one strain each of C. guilliermondii, C. krusei, C. lusitaniae, C. pelliculosa, and C. pseudointermedia isolate were subject to PFGE karyotyping as described above. The genetic relatedness of the total 61 C. albicans strains are shown in Fig. 1, a dendrogram constructed based on UPGMA clustering analysis of the PFGE results. The $\mathrm{S}_{\mathrm{AB}}$ values of the $C$. albicans isolates ranged from 0.71 to 1.0 . There were six clusters (i-vi) 
showing fairly even distribution of the isolates. Subjects harbouring C. albicans belonging to cluster vi were significantly older than the corresponding subjects of cluster i (58.8 vs. 37.0 years, $P=0.032$, Bonferroni multiple comparison). Subjects harbouring $C$. albicans belonging to cluster $v$ had a) lower quantity of the yeast in oral rinse samples than subjects with cluster vi (mean Candida: 38 vs 4060 c.f.u./ml, $P=0.01$, Kruskal-Wallis test), or b) larger quantity of the yeast in full mouth supra-gingival plaque than subjects with cluster iv (mean Candida: $4.2 \times 10^{6}$ vs. $5.9 \times 10^{4}$ c.f.u. $/ g, P=0.047$, Bonferroni multiple comparison).

Using $\mathrm{S}_{\mathrm{AB}} \geq 0.85$ to identify subgroups with closely related genetic clonotypes or karyotypes, fourteen (78\%), and eight (80\%) of the identified Candida isolate pairs from pSS and sSS groups respectively were found to be closely related $(P<0.001$, Kruskal-Wallis test). One pSS sufferer had one isolate pair of PFGE identical $C$. parapsilosis while another pSS sufferer had an identical pair of $C$. tropicalis (Figure 2). Twenty $C$. albicans isolate pairs out of a total of 61 isolates showed close karyotypic or genetic identity $\left(\mathrm{S}_{\mathrm{AB}} \geq 0.85\right.$, Figure 1$)$. Out of these 20 pairs, seven and three isolate pairs from the pSS and sSS groups respectively were karyotypically identical, i.e. $\mathrm{S}_{\mathrm{AB}}=1.0(P=0.008$, Kruskal-Wallis test $)$. One isolate pair each of C. parapsilosis, C. tropicalis, S. cerevisiae and T. mucoides isolates showed close or identical karyotypes $\left(\mathrm{S}_{\mathrm{AB}} \geq 0.85\right)$. One pSS subject had two karyotypically identical isolate pairs, i.e. one pair each of $C$. albicans and T. mucoides. The rest of the yeast isolates did not appeared to be karotypically related to each other. In summary, 22 of 52 SS subjects $(42 \%)$ had genetically related Candida or yeasts strains isolatable from oral rinse sample and supra-gingival plaque at the same time, 5 more subjects had positive Candida isolation from both oral rinse and supra-gingival plaque but were apparently not genetically related; another SS subject had unrelated $C$. albicans isolate-pair which the yeasts were genetically related to isolates from 2 other SS individuals; 5 or 7 subjects had single Candida species isolated only from oral rinse or plaque, respectively. 
Among SS subjects with a C. albicans isolate pair, their oral mucosa (as detected by oral rinse) and full mouth supra-gingival plaque were apparently colonized by a genetically related, if not identical, strain of the same yeast (C. albicans isolates form isolate pairs or not vs $\mathrm{S}_{\mathrm{AB}}<0.85$ or $\mathrm{S}_{\mathrm{AB}} \geq 0.85 ; P<0.001$, Fisher exact test). They also showed a higher but not statistically significant mean Candida count than individuals with no C. albicans isolate pair detectable (1124 cfu vs $44 \mathrm{cfu})$.

One representative isolate pair from each of the 5 C. albicans karyotypic clusters (except cluster v which lacks isolate pair), and one each of C. parapsilosis, C. tropicalis, S. cerevisiae and T. mucoides isolate pair are shown (Fig. 2).

\section{Discussion}

The prevalence of clinically detectable oral candidiasis among SS sufferers in the present study was around $20 \%(\mathrm{n}=10)$ which is at the lower end of the reported range. The low rate of oral candidiasis observed in the current study is possibly due to good oral and/or denture hygiene and the stringent diagnostic criteria for oral candidiasis used (Soto-Rojas et al, 1998). The current study revealed that oral candidiasis in the present cohort of regularly dentally cared for SS sufferers seemed to be associated with the presence of yeast in full mouth supra-gingival plaque. The current understanding that Candida residing in oral biofilm as one determinant of the agent in oral mycotic infection (Coogan et al. 2006) provided theoretical support for this observation. The fact that no swab or imprint sample was taken at candidiasis sites apparently prevented the direct comparison between the yeast causing the infection and those residing in supra-gingival plaque. Hence one must consider this limitation when interpreting the current data. It is known however, that the turnover rate for the intact oral epithelium layer is $4-14$ days (Squier et al, 1976) implying the quick exfoliation of the 
superficial cell layers. Therefore, for successful colonization by yeasts, a recurring source of "pioneering micro-colonies of yeasts" from an already established biofilm reservoir would be pivotal.

Although many sampling methods for oral mucosal flora have been devised it is now generally recognised that the oral rinse sampling method designed by Samaranayake et al, (1986) two decades ago is the simplest and practical method for the purpose, especially when focal erythematous or pseudo-membranous lesions are unapparent. Furthermore, the employment of this standard methodology provides a comparator useful for relative assessment between different studies conducted in the past for normal, non-SS (Leung et al, 2003), SS (Kindelan et al, 1998; Radfar et al, 2003), or other xerostomic subjects (Leung et al, 2000).

No report, however, is available reporting how often and how many "pioneering micro-colonies" would be released from supra-gingival plaque per unit time $(60 \mathrm{~s}$ in the current case) after oral rinse. On the other hand, considering the subjects in this study were of generally good oral hygiene (up to $90 \%$ tooth sites without visible plaque) and hence with small amount of supra-gingival plaque and thus the total surface area of oral mucosa exposed to oral rinse sampling is substantially greater than the supra-gingival plaque, we postulated that our oral rinse sampling "predominantly detected the mucosal colonization of oral yeast" among the subjects surveyed in this particular situation.

It was in only one sSS subjects that negative oral rinse and supra-gingival plaque culture was associated with clinical candidasis. Perhaps in such subject the infection was associated with yeasts at below the detection level. Another SS subject was classified as suffering from candidiasis because of angular cheilitis at the left corner of mouth. The same subject had otherwise normal intact oral mucosa. Failure of yeast detection in that individual was perhaps due to inability of the oral rinse sample to reach corners of mouth, or again the offending 
yeast was at a level below our detection limit, or the possibility that non-mycotic infectious agent might be involved (Dias \& Samaranayake, 1995). There is no guideline available regarding the critical yeast count above which clinical signs of oral candidiasis will be evident, although it is generally agreed that oral candidiasis is observed in individuals with high yeast counts, e.g. reported median c.f.u. of 1349 as measured by oral rinse or swab (Abraham et al, 1998). Comparing the reported data to our current low median level of 280 c.f.u./ml oral rinse in regularly dentally cared SS subjects with candidiasis, one would readily appreciate why in a small proportion of the affected individual we failed to detect the presence of yeast. The corresponding yeast levels from oral rinse in candidiasis or non-candidiasis SS subjects were not significantly different might also help explain such observation. In a cohort of elderly institutionalized people with poor oral hygiene and $19 \%$ of them xerostomic, Grimoud et al (2005) showed that after a 3-month oral hygiene programme, the prevalence of oral candidiasis and total quantity of yeasts reduced measurably reflecting that oral yeast colonization in susceptible subjects could be reduced by attention to oral cleanliness. The current SS sufferers and controls, however, demonstrated reasonable oral hygiene with up to $90 \%$ tooth sites without visible plaque. Despite the similar levels of oral hygiene, the prevalence of yeast colonization in controls was only detectable from oral rinse samples at a low level (7\%) while the corresponding prevalence were $\geq 50 \%$ for SS sufferers. In order to establish a clearer linkage between clinical candidiasis, oral hygiene and oral yeast colonization, a longitudinal investigation, involving a larger sample size, correlating the microbiology, presence or absence of oral candidiasis vs plaque control as well as supra-gingival plaque yeast prevalence or counts would be needed.

Only two out of eight pSS denture wearers (25\%) surveyed had oral candidiasis, one of whom had a denture hygiene score of 2 . In contrast to common belief, candidiasis did not appear to be associated with removable denture wearing in the present SS cohort who 
incidentally had reasonable denture hygiene and were free of denture-related stomatitis. Such negative findings between candidiasis and denture wearing are supported by Soto-Rojas et al (1998).

In the current study, prevalence of yeast colonization was $84 \%$ and $63 \%$ respectively for pSS and sSS groups which is significantly higher than controls (7\%). This finding compares favourably with a number of similar studies which reported $59 \%$ to almost $100 \%$ yeast colonization in SS sufferers (Tapper-Jones et al, 1980; Lundström \& Lindström, 1995; Abraham et al, 1998, Radfar et al, 2003). The increased rate of yeast colonization is considered to be the effect of hyposalivation as significantly lower salivary flow rates were observed.

Two previous reports (Kindelan et al, 1998; Pedersen et al, 1999) have demonstrated a higher rate of yeast colonization in primary compared with secondary SS sufferers which was also observed in the current study. However, previous studies did not explain the observation. A lower, but not statistically significant, salivary flow rate has been uniformly detected in pSS sufferers compared to sSS subjects (Daniels et al, 1975; van der Reijden et al, 1996; Almståhl et al, 1999). It is postulated that two factors may account for the higher rate of yeast colonization in the pSS group, i.e. reduced saliva production and/or denture wearing. The fact that none of our sSS subjects were denture wearers limited our ability to genuinely substantiate the impact of reduced saliva production and yeast colonization among pSS or sSS subjects.

All SS denture wearers in this study had positive yeast cultures for both oral rinse and plaque samples. A similar observation was also reported by Radfar et al (2003), although Tapper-Jones et al (1980) reported a slightly lower figure. In the present study, a strong correlation between removable denture wearing and yeast colonization was detected which had not been reported previously in SS patients. Yet, this finding should be interpreted with 
caution as there were only eight SS denture wearers involved. Nonetheless, removable prostheses wearing has been long recognized as a risk factor for yeast carriage as the effect of diminished salivary flow in SS subjects on yeast growth is further augmented by removable denture wearing. Oral prostheses are believed to cause persistent trauma to the oral mucosa and promote proliferation of yeast (Nyquist, 1992). They also provide microscopically rough surface for the yeast to inhabit (Taylor et al, 1998).

Using the oral rinse sampling technique, Candida counts of pSS sufferers have been reported to be around 1000 c.f.u./ml by a number of investigators and is significantly different to healthy controls (Abraham et al, 1998; Kindelan et al, 1998; Almståhl et al, 1999). Our results compare favourably with previous work. A lower mean yeast count was obtained from sSS sufferers, which supports the findings of some researchers (Kindelan et al, 1998; Rhodus et al, 1998) but not others (Rhodus et al, 1997; Almståhl et al, 1999). To our knowledge, there is only one study which has reported the amount of $C$. albicans in the supra-gingival plaque of pSS subjects (Almståhl et al, 2001). They reported 60\% and 25\% of pSS and controls respectively had $C$. albicans in supra-gingival plaque samples, and a significantly higher mean $C$. albicans count in pSS than controls. However, a significant proportion of their cohort frequently used saliva-stimulant which has been shown to lead to an increased salivary flow rate and a decreased amount of $C$. albicans colonization (Rhodus et al, 1998), making direct comparison with the current report difficult. Nonetheless, it was noteworthy that the mean C. albicans counts (c.f.u./g wet weight plaque) of the present pSS cohort was deduced to be almost 2000 -fold that obtained by the Swedish group while the mean proportion viable count of $C$. albicans in pSS non-denture wearers was 100-fold less than the Swedish report (0.0003\% vs 0.04\%) (Almståhl et al, 2001).

Among the yeast species identified, the majority were $C$. albicans which is in agreement with many other investigations (Hernandez \& Daniels, 1989; Kindelan et al, 1998; 
Soto-Rojas et al, 1998; Torres et al, 2002; Radfar et al, 2003). This can be explained by the fact that $C$. albicans are more robust and can survive and adapt to diverse environments by phenotypic and/or genotypic switching (Cannon et al, 1995; Leung et al, 2000). Moreover, six SS subjects from each group (24\% pSS and 22\% sSS) showed mixed colonization which compares favourably with the $28 \%$ noted by Torres et al (2002). Mixed colonization has also been observed, though less frequently, in patients with HIV (Tsang \& Samaranayake, 2000) and advanced malignancy (Jobbins et al, 1992). It is mostly due to alteration in host defence which promotes colonization by other non- $C$. albicans opportunistic pathogens such as $C$. dubliniensis, C. krusei and C. parapsilosis (Weems, 1992; Samaranayake \& Samaranayake, 1994; Gutierrez et al, 2002). Furthermore, the use of CHROMagar Candida as the isolation medium has been shown to have high sensitivity and specificity in detection of mixed cultures (Kindelan et al, 1998; Willinger \& Manafi, 1999).

To our knowledge, this is the first time that oral Candida colonization has been characterized and studied from oral rinse and full mouth supra-gingival plaque samples of SS sufferers. A variety of molecular based techniques are available including electrophoretic karyotyping, restriction fragment length polymorphisms (RFLP) (Merz 1990, review), randomly amplified polymorphic DNA (RAPD) (Leung et al, 2000; Dassanayake and Samaranayake 2003, review), and other PCR based approaches such as repetitive sequence-based PCR (rep-PCR) (Chen et al, 2005). High variability or low reproducibility are frequently encountered using RFLP or RAPD particularly under low-stringency conditions (Leung et al, 2000; Dassanayake and Samaranayake 2003) while the rep-PCR technique appears to rectify, to some extent, the short fall of PCR based techniques (Chen et al, 2005). The PCR or the RFLP based methods gave high discriminatory indices and were effective in discriminating particular pathogenic strain of the yeast from regular isolates (Chen et al, 2005). On the other hand, to identify clonal relatedness of isolates from the same 
host, electrophoretic karyotyping appeared to be among the best approaches (Leung et al, 2000; Chen et al, 2005) and was thus used in the current study.

Using electrophoretic karyotyping, we were able to show that out of the 52 SS sufferers, $22(42 \%)$ had genetically related Candida or yeast strains colonizing oral mucosa and supra-gingival plaque at the same time. Comparing genetically related C. albicans isolation pairs to the other $C$. albicans strains obtained from the same cohort, interesting findings were observed. No two subjects were colonized by C. albicans with same PFGE genetic identity, although 10 subjects colonized by C. albicans strains showed PFGE genetic relatedness to a C. albicans strain from one other individual among their group of which the genetic relatedness detected by PFGE (mean $\mathrm{S}_{\mathrm{AB}} \pm \mathrm{SD}=0.87 \pm 0.02$ ) was smaller than that between the C. albicans isolate pair from the same individual $\left(\mathrm{S}_{\mathrm{AB}}=0.94 \pm 0.07, P=0.0003\right.$, unpaired t test with Welch correction). We postulated that the $C$. albicans recovered from the SS subjects most likely had diverse origins.

In the majority of the subjects the oral mucosa and supra-gingival plaque were colonized by closely genetically related or identical yeast strains which lead us to postulate that persistent oral mucosal yeast infections might be aggravated by supra-gingival plaque colonization of the offending yeast or its genetically related form. The fact that sessile rather than planktonic C. albicans is more resistant to antifungal in vitro (Ramage et al, 2001) favours the notion that the yeast would be in a protected, stable environment within the plaque biofilm. The yeast might, from time to time be released by the biofilm and cause mucosal infection in those who were affected. The observation that Candida colonization of the gastrointestinal tract originated from carious teeth which constituted an ecologic niche (Hossain et al, 2003), tends to support this hypothesis albeit indirectly. Further investigations, however, are needed to establish the above hypothesis. We postulate that SS sufferers who had supra-gingival plaque colonized with yeast or Candida species might be more readily 
affected by mucosal colonization of the same or genetically related yeast species.

No Candida was recovered in the full mouth supra-gingival plaque samples of the ageand gender-matched controls but was detectable at high prevalence among all SS sufferers, despite more pSS subjects being denture wearers. This implies that Candida is able to establish itself in the supra-gingival plaque, a natural biofilm, of SS sufferers even if they practise good oral hygiene as was the case in our current cohort. Whether this establishment is due to colonization by biofilm-forming $C$. albicans strains in $\mathrm{SS}$, or due to diminished salivary flow rate and defence mechanisms leading to reduced selection pressure against $C$. albicans, or both, needs to be further explored. However, a recent in-vitro study found no quantitative differences in biofilm formation ability between C. albicans from HIV-infected versus HIV-free individuals on a micro-titre plate surface (Jin et al, 2003) possibly favouring the latter contention. Nonetheless, the clinical implication of such a finding is that once Candida is established in plaque, clinical and subclinical oral candidiasis might become persistent and difficult to manage, as treatment might involve not only topical application of antifungal agents to the oral mucosa but also an exceptionally high standard of plaque control. The reason being as in the case of the current SS subjects, they had C. albicans established in supra-gingival plaque despite good oral hygiene and high dental awareness (Leung et al, 2004). Another concern is that Candida colonization of supra-gingival plaque can also serve as a reservoir for disseminated infections such as aspiration pneumonia and gastrointestinal infections in compromised individuals (Cannon et al, 1995; Hossain et al, 2003).

Within the limitations of the present study, Chinese SS sufferers with good oral hygiene appeared to be prone to oral Candida colonization. Clinical oral candidiasis was only relatively commonly detected. C. albicans was the predominant oral yeast isolated. Candida species were also found to be established in the supra-gingival plaque of many of the SS sufferers. 


\section{Acknowledgements}

The work described in this paper was funded by grants from the Committee of Research and Conference Grants of the University of Hong Kong (WKL, 10202569; KCML, 10203765). We would also like to thank Chak S. Lau and Temy M.Y. Mok for access to the SS patients attending the Queen Mary Hospital, Hong Kong SAR, China.

\section{References}

Abraham CM, al-Hashimi I, Haghighat, N (1998). Evaluation of the levels of oral Candida in patients with Sjögren's syndrome. Oral Surg Oral Med Oral Pathol Oral Radio Endod 86: $65-68$.

Almståhl A, Kroneld U, Tarkowski A, Wikström M (1999). Oral microbial flora in Sjögren's syndrome. J Rheumatol 26: 110-114.

Almståhl A, Wikström M, Kroneld U (2001). Microflora in oral ecosystems in primary Sjögren's syndrome. J Rheumatol 28: 1007-1013.

Baudet-Pommel M, Albuisson E, Kemeny JL et al (1994). Early dental loss in Sjögren's syndrome. Histologic correlates. European Community Study Group on Diagnostic Criteria for Sjögren's syndrome (EEC COMAC). Oral Surg Oral Med Oral Pathol 78: 181-186.

Blair Y, Bagg J, MacFarlane TW, Chestnutt I (1995). Microbiological assessment of denture hygiene among patients in longstay and daycare community places. Community Dent Oral Epidemiol 23: 100-103.

Cannon RD, Holmes AR, Mason AB, Monk BC (1995). Oral Candida: clearance, 
colonization, or candidiasis. J Dent Res 74: 1152-1161.

Chen K-W, Lo H-J, Lin Y-H, Li S-Y (2005). Comparison of four molecular typing methods to assess genetic relatedness of Candida albicans clinical isolates in Taiwan. $J$ Med Microbiol 54: 249-258.

Clemons KV, Feroze F, Holmberg K, Stevens DA (1997). Comparative analysis of genetic variability among Candida albicans isolates from different geographic locales by three genotypic methods. J Clin Microbiol 35: 1332-1336.

Coogan MM, Fidel PL Jr, Komesu MC, Maeda N, Samaranayake LP (2006). (B1) Candida and mycotic infections. Adv Dent Res 19: 130-138.

Daniels TE, Silverman SJr, Michalski JP, Greenspan JS, SylvesterRA, Talal N (1975). The oral component of Sjögren's syndrome. Oral Surg 39: 875-885.

Dassanayake RS, Samaranayake LP (2003). Amplification-based nucleic acid scanning techniques to assess genetic polymorphism. Cirt Rev Microbiol 29: 1-24.

Dias AP, Samaranayake LP (1995). Clinical, microbiological and ultrastructural features of angular cheilitis lesions in southern Chinese. Oral Dis 1: 43-48.

Fox RI (2003). Sjögren's syndrome: evolving therapies. Expert Opin Investig Drugs 12: 247-254.

Grimoud AM, Lodter JP, Marty N et al (2005). Improved oral hygiene and Candida species colonization level in geriatric patients. Oral Dis 11: 163-169.

Gutierrez J, Morales P, Gonzalez MA, Quindos G (2002). Candida dubliniensis, a new fungal pathogen. J Basic Microbiol 42: 207-227.

Hernandez YL, Daniels TE (1989). Oral candidiasis in Sjögren's syndrome: prevalence, clinical correlations, and treatment. Oral Surg Oral Med Oral Pathol 68: 324-329.

Hossain H, Ansari F, Schulz-Weidner N, Wetzel WE, Chakraborty T, Domann E (2003). Clonal identity of Candida albicans in the oral cavity and the gastrointestinal tract of 
pre-school children. Oral Microbiol Immunol 18: 302-308.

Jin Y, Yip HK, Samaranayake YH, Yau JY, Samaranayake LP (2003). Biofilm-forming ability of Candida albicans is unlikely to contribute to high levels of oral yeast carriage in cases of human immunodeficiency virus infection. J Clin Microbiol 41: 2961-2967.

Jobbins J, Bagg J, Parsons K, Finlay I, Addy M, Newcombe RG (1992). Oral carriage of yeasts, coliforms and staphylococci in patients with advanced malignant disease. J Oral Pathol Med 21: 305-308.

Kindelan SA, Yeoman CM, Douglas CWI, Franklin C (1998). A comparison of intraoral Candida carriage in Sjögren's syndrome patients with healthy xerostomic controls. Oral Surg Oral Med Oral Pathol Oral Radiol Endod 85: 162-167.

Leung KCM, McMillan AS, Leung WK, Wong MCM, Lau CS, Mok TMY (2004). Oral health condition and saliva flow in southern Chinese with Sjögren's syndrome. Int Dent J 54: 159-165.

Leung WK, Dassanayake RS, Yau JYY, Jin LJ, Yam WC, Samaranayake LP (2000). Oral colonization, phenotypic, and genotypic profiles of Candida species in irradiated, dentate, xerostomic nasopharyngeal carcinoma survivors. J Clin Microbiol 38: 2219-2226.

Leung WK, Jin L, Yau JYY, Sun Q, Corbet EF (2005). Microflora cultivable from minocycline strips placed in persisting periodontal pockets. Arch Oral Biol 50:39-48.

Leung WK, Yau JYY, Cheung BPK, Jin LJ, Zee K-Y, Lo ECM, Samaranayake LP, Corbet EF (2003). Oral colonisation by aerobic and facultatively anaerobic gram-negative rods and yeast in Tibetans living in Lhasa. Arch Oral Biol 48:117-23.

Löe H, Silness J (1963). Periodontal disease in pregnancy. I. Prevalence and severity. Acta Odont Scand 21:533-551.

Lundström IMC, Lindström FD (1995). Subjective and clinical oral symptoms in patients with primary Sjögren's syndrome. Clin Exp Rheumatol 13: 725-731. 
McMillan AS, Leung KCM, Leung WK, Wong MCM, Lau CS, Mok TMY (2004). Impact of Sjögren's syndrome on oral health-related quality of life in southern Chinese. J Oral Rehabil 31: 653-659.

Merz WG (1990). Candida albicans strain delineation. Clin Microbiol Rev 3: 321-334.

Newton AV (1962). Denture sore mouth: a possible aetiology. Br Dent J 112: 357-360.

Nyquist G (1992). A study of denture sore mouth. An investigation of traumatic, allergic and toxic lesions of the oral mucosa arising from the use of full dentures. Acta Odont Scand 10: Suppl 9, 11-14.

Pedersen AM, Reibel J, Nordgarden H, Bergem HO, Jensen JL, Nauntofte B (1999). Primary Sjögren's syndrome: salivary gland function and clinical oral findings. Oral Dis 5: $128-138$.

Radfar L, Shea Y, Fischer SH et al (2003). Fungal load and candidiasis in Sjögren's syndrome. Oral Surg Oral Med Oral Pathol Oral Radiol Endod 96: 283-287.

Ramage G, Vandewalle K, Wickes BL, Lopez-Ribot JL (2001). Characteristics of biofilm formation by Candida albicans. Rev Iberoam Micol 18:163-170.

Rhodus NL, Bloomquist C, Liljemark W, Bereuter J (1997). Prevalence, density, and manifestations of oral Candida albicans in patients with Sjögren's syndrome. $J$ Otolaryngol 26: 300-305.

Rhodus NL, Liljemark W, Bloomquist C, Bereuter J (1998). Candida albicans levels in patients with Sjögren's syndrome before and after long-term use of pilocarpine hydrochloride: a pilot study. Quintessence Int 29: 705-710.

Samaranayake LP, MacFarlane TW, Lamey PJ, Ferguson MM (1986). A comparison of oral rinse and imprint sampling techniques for detection of yeasts, coliform and Staphylococcus aureus in the oral cavity. J Oral Pathol 15: 386-388.

Samaranayake YH, Samaranayake LP (1994). Candida krusei: biology, epidemiology, 
pathogenicity and clinical manifestations of an emerging pathogen. $J$ Med Microbiol 41: 295-310.

Sancak B, Colakoglu S, Acikgoz ZC, Arikan S (2005). Incubation at room temperature may be an independent factor that induces chlamydospore production in Candida dubliniensis. Diagn Microbiol Infect Dis 52: 305-309.

Silness J, Löe H (1964). Periodontal disease in pregnancy: II Correlation between oral hygiene and periodontal condition. Acta Odont Scand 22: 121-135.

Soto-Rojas AE, Villa AR, Sifuentes-Osornio J, Alarćon-Segovia D, Kraus A (1998). Oral candidiasis and Sjögren's syndrome. J Rheumatol 25: 911-915.

Squier CA, Johnson NW, Hopps RM (1976). Human oral mucosa. Development, structure and function. Oxford: Blackwell Scientific Publications.

Tapper-Jones L, Aldred M, Walker DM (1980). Prevalence and intraoral distribution of Candida albicans in Sjögren's syndrome. J Clin Pathol 33: 282-287.

Taylor R, Maryan C, Verran J (1998). Retention of oral microorganisms on cobalt-chromium alloy and dental acrylic resin with different surface finishes. J Prosthet Dent 80: 592-597.

Torres SR, Peixoto CB, Caldas DM et al (2002). Relationship between salivary flow rates and Candida counts in subjects with xerostomia. Oral Surg Oral Med Oral Pathol Oral Radiol Endod 93: 149-154.

Tsang CS, Samaranayake LP (2000). Oral yeasts and coliforms in HIV-infected individuals in Hong Kong. Mycoses 43: 303-308.

van der Reijden WA, van der Kwaak JS, Veerman ECI, Nieuw Amerongen AV (1996). Analysis of the concentration and output of whole salivary constituents in patients with Sjögren's syndrome. Eur J Oral Sci 104: 335-340.

Vitali C, Bombardieri S, Jonsson R et al (2002). Classification criteria for Sjögren's syndrome: a revised version of the European criteria proposed by the American-European 
Consensus Group. Ann Rheum Dis 61: 554-558.

Willinger B, Manafi M (1999). Evaluation of CHROMagar Candida for rapid screening of clinical specimens for Candida species. Mycoses 42: 61-65.

Weems JJJr (1992). Candida parapsilosis: epidemiology, pathogenicity, clinical manifestations, and antimicrobial susceptibility. Clin Infect. Dis 14: 756-766. 
Table 1 Quantity and prevalence of yeast isolated from oral rinse and supra-gingival plaque samples

\begin{tabular}{|c|c|c|c|}
\hline & $\begin{array}{c}p S S \\
(n=25)\end{array}$ & $\begin{array}{c}s S S \\
(n=27)\end{array}$ & $\begin{array}{l}\text { Control } \\
(n=29)\end{array}$ \\
\hline$\%$ oral candidiasis $^{\mathrm{a}}$ & 20 & 18.5 & 0 \\
\hline \multicolumn{4}{|l|}{$\%$ yeast colonization } \\
\hline oral rinse $\mathrm{b}^{\mathrm{b}}$ & 72.0 & 48.1 & 6.9 \\
\hline plaque $^{\mathrm{b}}$ & 84.0 & 55.6 & 0.0 \\
\hline oral rinse and plaque ${ }^{b}$ & 72.0 & 40.7 & 0.0 \\
\hline$\%$ denture wearers with yeast colonization & $n=8$ & $n=0$ & $n=2$ \\
\hline oral rinse & 100.0 & $\mathrm{NA}^{\mathrm{c}}$ & 50.0 \\
\hline plaque $^{\mathrm{d}}$ & 100.0 & NA & 0.0 \\
\hline oral rinse and plaque ${ }^{d}$ & 100.0 & NA & 0.0 \\
\hline \multicolumn{4}{|l|}{ yeast count (mean $\pm \mathrm{SD})$} \\
\hline oral rinse (c.f.u./ml) ${ }^{\mathrm{e}}$ & $1025.5 \pm 3882.1$ & $155.0 \pm 327.2$ & $2.1 \pm 8.2$ \\
\hline denture wearers & $2689.8 \pm 6836.3$ & NA & $20.0 \pm 28.3$ \\
\hline non-denture wearers ${ }^{\mathrm{e}}$ & $242.4 \pm 351.3$ & $155.0 \pm 327.2$ & $0.7 \pm 3.9$ \\
\hline plaque $\left(\times 10^{6} \text { c.f.u. } / \mathrm{g}\right)^{\mathrm{e}}$ & $1.8 \pm 4.1$ & $0.4 \pm 0.8$ & 0.0 \\
\hline denture wearers & $0.9 \pm 1.0$ & NA & 0.0 \\
\hline non-denture wearers ${ }^{\mathrm{e}}$ & $2.3 \pm 5.0$ & $0.4 \pm 0.8$ & 0.0 \\
\hline \multicolumn{4}{|l|}{ Candida count (mean $\pm \mathrm{SD})$} \\
\hline oral rinse (c.f.u./ml) ${ }^{\mathrm{e}}$ & $1017.5 \pm 3883.7$ & $149.1 \pm 322.7$ & $2.1 \pm 8.2$ \\
\hline plaque $\left(\times 10^{6} \text { c.f.u. } / \mathrm{g}\right)^{\mathrm{e}}$ & $1.8 \pm 4.1$ & $0.4 \pm 0.8$ & 0.0 \\
\hline \multicolumn{4}{|l|}{$\begin{array}{l}\% \text { per total anaerobic viable count in } \\
\text { supra-gingival plaque }\end{array}$} \\
\hline yeast $\left(\times 10^{-3}\right)^{\mathrm{e}}$ & $0.4 \pm 0.7$ & $1.2 \pm 5.2$ & 0.0 \\
\hline denture wearers & $0.5 \pm 0.5$ & NA & 0.0 \\
\hline non-denture wearers ${ }^{\mathrm{e}}$ & $0.3 \pm 0.7$ & $1.2 \pm 5.2$ & 0.0 \\
\hline Candida $\left(\times 10^{-3}\right)^{\mathrm{e}}$ & $0.4 \pm 0.7$ & $1.1 \pm 4.8$ & 0.0 \\
\hline
\end{tabular}

${ }^{\mathrm{a}} P=0.012$, Fisher's exact test, pSS and sSS subjects grouped together.

${ }^{\mathrm{b}}$ Significant difference among the groups, $P<0.0001, \chi^{2}$ test.

${ }^{\mathrm{c}} \mathrm{NA}=$ not applicable.

${ }^{\mathrm{d}} P=0.022$, Fisher's exact test; no removable denture wearer in sSS group.

eSignificant difference among the groups, $P<0.001$, Kruskal-Wallis test. 
Table 2 Identity, frequency and quantity of cultivable yeast isolated from oral rinse samples and full mouth supra-gingival plaque ${ }^{\mathrm{a}}$.

\begin{tabular}{|c|c|c|c|c|c|c|c|c|c|c|}
\hline & \multicolumn{6}{|c|}{ Oral rinse } & \multicolumn{4}{|c|}{ Supra-gingival plaque $^{b}$} \\
\hline & $(n=$ & & \multicolumn{2}{|c|}{$\begin{array}{c}s S S \\
(n=27)\end{array}$} & \multicolumn{2}{|c|}{$\begin{array}{l}\text { Control } \\
(n=29)\end{array}$} & \multicolumn{2}{|c|}{$\begin{array}{c}p S S \\
(n=25)\end{array}$} & \multicolumn{2}{|c|}{$\begin{array}{c}s S S \\
(n=27)\end{array}$} \\
\hline & Prevalence & $\begin{array}{c}\text { Mean } \\
\text { viable } \\
\text { count } \\
\text { (c.f.u./ml) }\end{array}$ & Prevalence & $\begin{array}{c}\text { Mean } \\
\text { viable } \\
\text { count } \\
\text { (c.f.u./ml) }\end{array}$ & $\begin{array}{c}\text { Mean } \\
\text { viable } \\
\text { count } \\
\text { (c.f.u. } / m l)\end{array}$ & $\begin{array}{c}\text { Mean } \\
\text { viable } \\
\text { count } \\
\text { (c.f.u./ml) }\end{array}$ & Prevalence & $\begin{array}{c}\text { Mean } \\
\text { viable } \\
\text { count } \\
\text { (c.f.u. } / g \text { ) }\end{array}$ & Prevalence & $\begin{array}{l}\text { Mean } \\
\text { viable } \\
\text { count } \\
\text { (c.f.u./g) }\end{array}$ \\
\hline \multicolumn{11}{|l|}{ Candida } \\
\hline Candida albicans ${ }^{\mathrm{c}}$ & $16(64 \%)$ & 1015.1 & $12(44 \%)$ & 149.1 & 0 & 0 & $18(72 \%)$ & $18 \times 10^{5}$ & $15(56 \%)$ & $4 \times 10^{5}$ \\
\hline Candida dubliniensis & 0 & 0 & 0 & 0 & 0 & 0 & $1(4 \%)$ & 5846 & $1(4 \%)$ & 898 \\
\hline Candida glabrata & $2(8 \%)$ & 1.6 & 0 & 0 & 0 & 0 & 0 & 0 & 0 & 0 \\
\hline Candida guilliermondii & 0 & 0 & 0 & 0 & $1(3 \%)$ & 1.4 & 0 & 0 & 0 & 0 \\
\hline Candida krusei & 0 & 0 & 0 & 0 & $1(3 \%)$ & 0.7 & 0 & 0 & 0 & 0 \\
\hline Candida lusitaniae & 0 & 0 & $1(4 \%)$ & 0.7 & 0 & 0 & 0 & 0 & 0 & 0 \\
\hline Candida parapsilosis & $2(8 \%)$ & 2.4 & $2(7 \%)$ & 1.5 & 0 & 0 & $1(4 \%)$ & 143 & 0 & 0 \\
\hline Candida pelliculosa & 0 & 0 & $1(4 \%)$ & 1.5 & 0 & 0 & 0 & 0 & 0 & 0 \\
\hline Candida pseudointermedia & 0 & 0 & $1(4 \%)$ & 0.7 & 0 & 0 & 0 & 0 & 0 & 0 \\
\hline Candida tropicalis & $1(4 \%)$ & 2.4 & $1(4 \%)$ & 0.7 & 0 & 0 & $2(8 \%)$ & $1.7 \times 10^{4}$ & 0 & 0 \\
\hline \multicolumn{11}{|l|}{ Non-Candida } \\
\hline Saccharomyces cerevisiae & $1(4 \%)$ & 1.6 & $1(4 \%)$ & 0.7 & 0 & 0 & 0 & 0 & $1(4 \%)$ & 6173 \\
\hline Trichosporon mucoides & $1(4 \%)$ & 2.4 & 0 & 0 & 0 & 0 & $1(4 \%)$ & 1739 & 0 & 0 \\
\hline
\end{tabular}

${ }^{\mathrm{a}}$ Some individuals carried more than a single yeast species.

${ }^{b}$ No yeast was detected from full mouth supra-gingival plaque of control subjects $(n=29)$.

${ }^{\mathrm{c}}$ Significant difference regarding c.f.u. counts (per $\mathrm{ml}$ oral rinse or per gram wet weight supra-gingival plaque) among the groups, $P<0.001$, Kruskal-Wallis test. 


\section{LEGEND}

Fig. 1. Dendrogram showing the genetic divergence of 61 strains of $C$. albicans isolated from oral rinse (R) and full mouth supra-gingival plaque (P) of pSS (p) and sSS (s) subjects analyzed by pulse-field gel electrophoresis (PFGE). The dendrogram was constructed by unweighed pair group method with arithmetic means (UPGMA clustering analysis). A dashed line is drawn at $\mathrm{S}_{\mathrm{AB}}=0.85$ to denote the threshold selected to demarcate genetic relatedness of isolates (Clemons et al, 1997). Note that the C. albicans isolates formed 6 PFGE karotype clusters $\left(\mathrm{S}_{\mathrm{AB}} \geq 0.73\right.$, clusters $\left.\mathrm{i}-\mathrm{vi}\right)$. Ten isolate pairs of $C$. albicans ( 7 and 3 pairs from $\mathrm{pSS}$ and $\mathrm{sSS}$ respectively) were karotypically/genetically identical, i.e. $\mathrm{S}_{\mathrm{AB}}=1.0$; another 10 isolate pairs appeared to be genetically related. Representative C. albicans PFGE patterns are shown in Fig. 2.

Fig. 2. Representative PFGE separation of chromosome-sized DNA of yeast isolate pairs of different karyotypic clonal types from oral rinse samples and full mouth supra-gingival plaque from subjects with SS. Lane M1: Saccharomyces cerevisiae chromosome molecular size standard (Bio-Rad); lanes 1, 3, 5, 7, 9: C. albicans strains isolated from oral rinse samples; lanes 2, 4, 6, 8, 10: C. albicans strains isolated from full mouth supra-gingival plaque sample of the corresponding subjects; lanes 11 and 12, 13 and 14, 15 and 16, 17 and 18: C. tropicalis, C. parapsilosis, S. cerevisiae, or T. mucoides isolate pairs from oral rinse sample and full mouth supra-gingival plaque from the same subject, respectively; lane M2: Hansenula wingei chromosome molecular size standard (Bio-Rad). All isolate pairs except lanes 5 and 6,15 and $16(\mathrm{SAB} \geq 0.86)$ were found to be karotypically identical i.e. $\mathrm{S}_{\mathrm{AB}}=1.0$. 


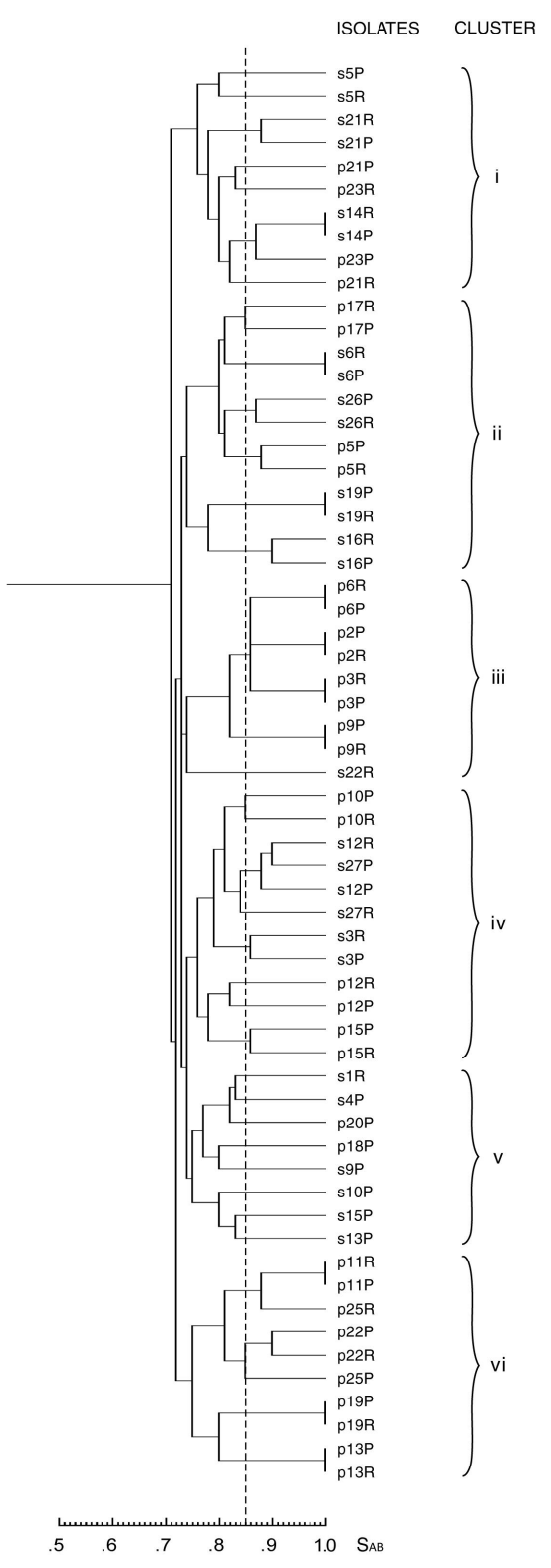

Fig. 1. Dendrogram showing the genetic divergence of 61 strains of $C$. albicans isolated from oral rinse (R) and full mouth supra-gingival plaque (P) of pSS (p) and sSS (s) subjects analyzed by pulse-field gel electrophoresis (PFGE). The dendrogram was constructed by unweighed pair group method with arithmetic means (UPGMA clustering analysis). A dashed line is drawn at $\mathrm{SAB}=0.85$ to denote the threshold selected to demarcate genetic relatedness of isolates (Clemons et al, 1997). Note that the C. albicans isolates formed 6 PFGE karotype clusters (SAB iÝ 0.73 , clusters i-vi). Ten isolate pairs of C. albicans ( 7 and 3 pairs from pSS and sSS respectively) were karotypically/genetically identical, i.e. SAB = 1.0; another 10 isolate pairs appeared to be genetically related.

Representative C. albicans PFGE patterns are shown in Fig. 2. 


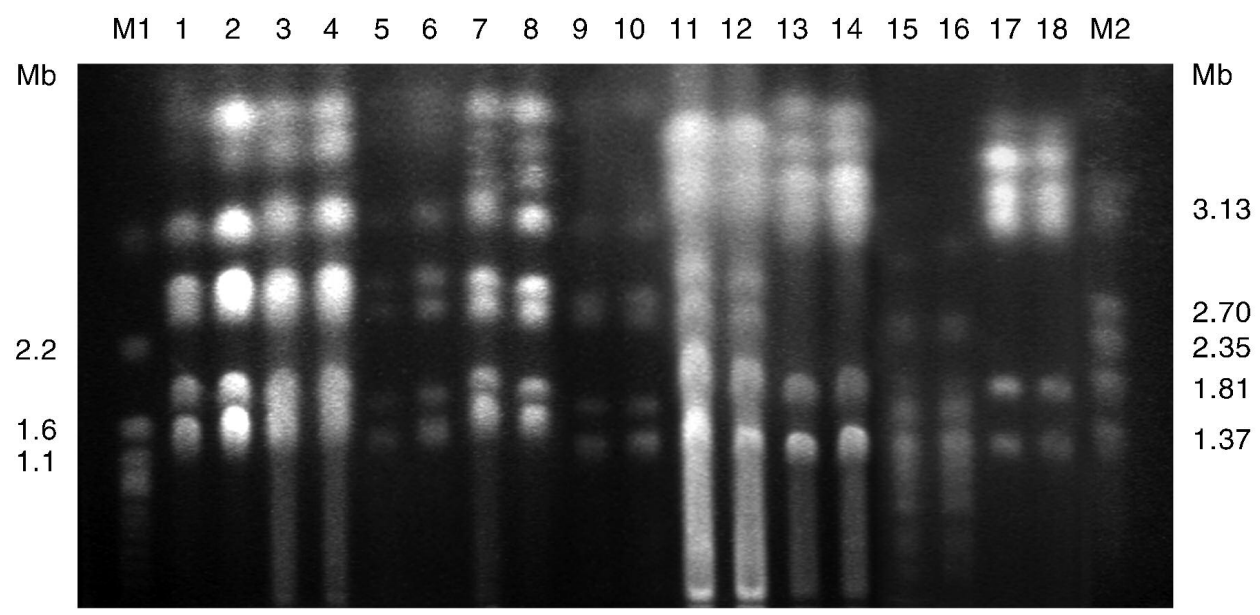

Fig. 2. Representative PFGE separation of chromosome-sized DNA of yeast isolate pairs of different karyotypic clonal types from oral rinse samples and full mouth supra-gingival plaque from subjects with SS. Lane M1: Saccharomyces cerevisiae chromosome molecular size standard (Bio-Rad); lanes 1, 3, 5, 7, 9: C. albicans strains isolated from oral rinse samples; lanes 2, 4, 6, 8, 10: C. albicans strains isolated from full mouth supra-gingival plaque sample of the corresponding subjects; lanes 11 and 12, 13 and 14, 15 and 16, 17 and 18: C. tropicalis, C. parapsilosis, S. cerevisiae, or T. mucoides isolate pairs from oral rinse sample and full mouth supra-gingival plaque from the same subject, respectively; lane M2: Hansenula wingei chromosome molecular size standard (Bio-Rad). All isolate pairs except lanes 5 and 6,15 and 16 (SAB iÝ 0.86) were found to be karotypically identical i.e. SAB $=1.0$. 